\title{
Pointwise approximation of modified
}

\section{conjugate functions by matrix operators of conjugate Fourier series of $2 \pi / r$-periodic functions}

Mateusz Kubiak, Włodzimierz Łenski and Bogdan Szal ${ }^{*}$

"Correspondence:

B.Szal@wmie.uz.zgora.pl

Faculty of Mathematics, Computer

Science and Econometrics,

University of Zielona Góra, Zielona

Góra, Poland

\begin{abstract}
We extend the results of Xh. Z. Krasniqi (Acta Comment. Univ. Tartu Math. 17:89-101, 2013) and the authors (Acta Comment. Univ. Tartu Math. 13:11-24, 2009; Proc. Est. Acad. Sci. 67:50-60, 2018) to the case when considered function is $2 \pi / r$-periodic and the measure of approximation depends on $r$-differences of the entries of the considered matrices.
\end{abstract}

MSC: $42 \mathrm{~A} 24$

Keywords: Rate of approximation; Summability of Fourier series

\section{Introduction}

Let $L_{2 \pi / r}^{p}(1 \leq p<\infty)$ be the class of all $2 \pi / r$-periodic real-valued functions, integrable in the Lebesgue sense with the $p$ th power over $Q_{r}=[-\pi / r, \pi / r]$ with the norm

$$
\|f\|_{L_{2 \pi / r}^{p}}=\|f(\cdot)\|_{L_{2 \pi / r}^{p}}:=\left(\int_{Q_{r}}|f(t)|^{p} d t\right)^{1 / p},
$$

where $r \in \mathbb{N}$. It is clear that $L_{2 \pi / r}^{p} \subset L_{2 \pi / 1}^{p}=L_{2 \pi}^{p}$ and for $f \in L_{2 \pi / r}^{p}$

$$
\|f\|_{L_{2 \pi}^{p}}=r^{1 / p}\|f\|_{L_{2 \pi / r}^{p}} .
$$

Taking into account the above relations, we will consider, for $f \in L_{2 \pi / r}^{1}$, the trigonometric Fourier series as such a series of $f \in L_{2 \pi}^{1}$ in the following form:

$$
S f(x):=\frac{a_{0}(f)}{2}+\sum_{v=1}^{\infty}\left(a_{v}(f) \cos v x+b_{v}(f) \sin v x\right)
$$

with the partial sums $S_{k} f$ and the conjugate one

$$
\widetilde{S} f(x):=\sum_{\nu=1}^{\infty}\left(a_{v}(f) \sin v x-b_{\nu}(f) \cos v x\right)
$$

(c) The Author(s) 2018. This article is distributed under the terms of the Creative Commons Attribution 4.0 International License (http://creativecommons.org/licenses/by/4.0/), which permits unrestricted use, distribution, and reproduction in any medium, provided you give appropriate credit to the original author(s) and the source, provide a link to the Creative Commons license, and indicate if changes were made. 
with the partial sums $\widetilde{S}_{k} f$. We also know that if $f \in L_{2 \pi}^{1}$, then

$$
\widetilde{f}(x):=-\frac{1}{\pi} \int_{0}^{\pi} \psi_{x}(t) \frac{1}{2} \cot \frac{t}{2} d t=\lim _{\epsilon \rightarrow 0^{+}} \tilde{f}(x, \epsilon)=\lim _{\epsilon \rightarrow 0^{+}} \tilde{f}_{r}(x, \epsilon),
$$

where, for $r \in \mathbb{N}$,

$$
\tilde{f}_{r}(x, \epsilon):= \begin{cases}-\frac{1}{\pi}\left(\sum_{m=0}^{[r / 2]-1} \int_{\frac{2 m \pi}{r}+\epsilon}^{\frac{2(m+1) \pi}{r}-\epsilon}+\int_{\frac{2[r / 2] \pi}{r}+\epsilon}^{\frac{(2[r / 2]+1) \pi}{r}}\right) \psi_{x}(t) \frac{1}{2} \cot \frac{t}{2} d t & \text { for an odd } r, \\ -\frac{1}{\pi} \sum_{m=0}^{[r / 2]-1} \int_{\frac{2 m \pi}{r}+\epsilon}^{\frac{2(m+1) \pi}{r}-\epsilon} \psi_{x}(t) \frac{1}{2} \cot \frac{t}{2} d t & \text { for an even } r\end{cases}
$$

and

$$
\widetilde{f}(x, \epsilon)=\widetilde{f}_{1}(x, \epsilon):=-\frac{1}{\pi} \int_{\epsilon}^{\pi} \psi_{x}(t) \frac{1}{2} \cot \frac{t}{2} d t,
$$

with

$$
\psi_{x}(t):=f(x+t)-f(x-t)
$$

exist for almost all $x$ (cf. [4, Th. (3.1) IV]).

Let $A:=\left(a_{n, k}\right)$ be an infinite matrix of real numbers such that

$$
a_{n, k} \geq 0 \quad \text { when } k, n=0,1,2, \ldots, \quad \lim _{n \rightarrow \infty} a_{n, k}=0 \quad \text { and } \quad \sum_{k=0}^{\infty} a_{n, k}=1,
$$

but $A^{\circ}:=\left(a_{n, k}\right)_{k=0}^{n}$, where

$$
a_{n, k}=0 \quad \text { when } k>n \text {. }
$$

We will use the notations

$$
A_{n, r}=\sum_{k=0}^{\infty}\left|a_{n, k}-a_{n, k+r}\right|, \quad A_{n, r}^{\circ}=\sum_{k=0}^{n}\left|a_{n, k}-a_{n, k+r}\right|
$$

for $r \in \mathbb{N}$ and

$$
\widetilde{T}_{n, A} f(x):=\sum_{k=0}^{\infty} a_{n, k} \widetilde{S}_{k} f(x) \quad(n=0,1,2, \ldots)
$$

for the $A$-transformation of $\widetilde{S} f$.

In this paper, we will study the estimate of $\left|\widetilde{T}_{n, A} f(x)-\widetilde{f}_{r}(x, \epsilon)\right|$ by the function of modulus of continuity type, i.e. a nondecreasing continuous function $\widetilde{\omega}$ having the following properties: $\widetilde{\omega}(0)=0, \widetilde{\omega}\left(\delta_{1}+\delta_{2}\right) \leq \widetilde{\omega}\left(\delta_{1}\right)+\widetilde{\omega}\left(\delta_{2}\right)$ for any $0 \leq \delta_{1} \leq \delta_{2} \leq \delta_{1}+\delta_{2} \leq 2 \pi$. We will also consider functions from the subclass $L_{2 \pi / r}^{p}(\widetilde{\omega})_{\beta}$ of $L_{2 \pi / r}^{p}$ for $r \in \mathbb{N}$ :

$$
L_{2 \pi / r}^{p}(\widetilde{\omega})_{\beta}=\left\{f \in L_{2 \pi / r}^{p}: \widetilde{\omega}_{\beta}(f, \delta)_{L_{2 \pi / r}^{p}}=O(\widetilde{\omega}(\delta)) \text { when } \delta \in[0,2 \pi] \text { and } \beta \geq 0\right\},
$$


where

$$
\widetilde{\omega}_{\beta} f(\delta)_{L_{2 \pi / r}^{p}}=\sup _{0 \leq|t| \leq \delta}\left\{\left|\sin \frac{r t}{2}\right|^{\beta}\|\psi \cdot(t)\|_{L_{2 \pi / r}^{p}}\right\} .
$$

It is easy to see that $\widetilde{\omega}_{0} f(\cdot)_{L_{2 \pi / r}^{p}}=\widetilde{\omega} f(\cdot)_{L_{2 \pi / r}^{p}}$ is the classical modulus of continuity. Moreover, it is clear that for $\beta \geq \alpha \geq 0$

$$
\widetilde{\omega}_{\beta} f(\delta)_{L_{2 \pi / r}^{p}} \leq \widetilde{\omega}_{\alpha} f(\delta)_{L_{2 \pi / r}^{p}}
$$

and consequently

$$
L_{2 \pi / r}^{p}(\widetilde{\omega})_{\alpha} \subseteq L_{2 \pi / r}^{p}(\widetilde{\omega})_{\beta} .
$$

The deviation $\widetilde{T}_{n, A} f(x)-\widetilde{f}_{r}(x, \epsilon)$ was estimated with $r=1$ in [2] and generalized in [1] as follows:

Theorem A ([1, Theorem 8, p. 95]) Iff $\in L_{2 \pi}^{p}(\widetilde{\omega})_{\beta}$ with $1<p<\infty$ and $0 \leq \beta<1-\frac{1}{p}$, where $\widetilde{\omega}$ satisfies the conditions:

$$
\left\{\int_{\frac{\pi}{n+1}}^{\pi}\left(\frac{t^{-\gamma}\left|\psi_{x}(t)\right|}{\widetilde{\omega}(t)}\right)^{p} \sin ^{\beta p} \frac{t}{2} d t\right\}^{1 / p}=O_{x}\left((n+1)^{\gamma}\right)
$$

with $0<\gamma<\beta+\frac{1}{p}$ and

$$
\left\{\int_{0}^{\frac{\pi}{n+1}}\left(\frac{t\left|\psi_{x}(t)\right|}{\widetilde{\omega}(t)}\right)^{p} \sin ^{\beta p} \frac{t}{2} d t\right\}^{1 / p}=O_{x}\left((n+1)^{-1}\right)
$$

then

$$
\left|\widetilde{T}_{n, A^{\circ}} f(x)-\widetilde{f}\left(x, \frac{\pi}{n+1}\right)\right|=O_{x}\left((n+1)^{\beta+\frac{1}{p}+1} A_{n, 1}^{\circ} \widetilde{\omega}\left(\frac{\pi}{n+1}\right)\right) .
$$

The next essential generalizations and improvements in [3, Theorem 1] were given. In these results $\widetilde{f}_{r}(x, \epsilon)$ and $A_{n, r}$ (with $r \in \mathbb{N}$ ) instead of $\widetilde{f}_{1}(x, \epsilon)=\widetilde{f}(x, \epsilon)$ and $A_{n, 1}^{\circ}$, respectively, were taken. We can formulate them as follows.

Theorem B ([3, Theorem 1]) If $f \in L_{2 \pi}^{p}, 1<p<\infty, 0 \leq \beta<1-\frac{1}{p}$ and a function $\widetilde{\omega}$ of modulus of continuity type satisfies the conditions:

$$
\left\{\int_{0}^{\frac{\pi}{r(n+1)}}\left(\frac{t \mid \psi_{x}(t) \| \sin \frac{r t}{2} \beta^{\beta}}{\widetilde{\omega}(t)}\right)^{p} d t\right\}^{1 / p}=O_{x}\left((n+1)^{-1}\right)
$$

for $r \in \mathbb{N}$,

$$
\left\{\int_{\frac{2 m \pi}{r}}^{\frac{2 m \pi}{r}+\frac{\pi}{r(n+1)}}\left(\frac{\left|\psi_{x}(t)\right|\left|\sin \frac{r t}{2}\right|^{\beta}}{\widetilde{\omega}\left(t-\frac{2 m \pi}{r}\right)}\right)^{p} d t\right\}^{1 / p}=O_{x}(1)
$$


for a natural $r \geq 3$, where $m \in\left\{1, \ldots\left[\frac{r}{2}\right]\right\}$ when $r$ is an odd or $m \in\left\{1, \ldots\left[\frac{r}{2}\right]-1\right\}$ when $r$ is an even natural number, and

$$
\left\{\int_{\frac{2 m \pi}{r}+\frac{\pi}{r(n+1)}}^{\frac{2 m \pi}{r}+\frac{\pi}{r}}\left(\frac{\left|\psi_{x}(t)\right|\left|\sin \frac{r t}{2}\right|^{\beta}}{\widetilde{\omega}(t)\left(t-\frac{2 m \pi}{r}\right)^{\gamma}}\right)^{p} d t\right\}^{1 / p}=O_{x}\left((n+1)^{\gamma}\right),
$$

for $r \in \mathbb{N}$ with $0<\gamma<\beta+\frac{1}{p}$, where $m \in\left\{0, \ldots\left[\frac{r}{2}\right]\right\}$ when $r$ is an odd or $m \in\left\{0, \ldots\left[\frac{r}{2}\right]-1\right\}$ when $r$ is an even natural number. Moreover, let $\widetilde{\omega}$ satisfy, for a natural $r \geq 2$, the conditions:

$$
\begin{aligned}
& \left\{\int_{\frac{2(m+1) \pi}{r}-\frac{\pi}{r(n+1)}}^{\frac{2(m+1) \pi}{\sigma}}\left(\frac{\left|\psi_{x}(t)\right|\left|\sin \frac{r t}{2}\right|^{\beta}}{\widetilde{\omega}\left(\frac{2(m+1) \pi}{r}-t\right)}\right)^{p} d t\right\}^{1 / p}=O_{x}(1), \\
& \left\{\int_{\frac{2(m+1) \pi}{r}-\frac{\pi}{r}}^{\frac{2(m+1) \pi}{r(n+1)}}\left(\frac{\left|\psi_{x}(t)\right|\left|\sin \frac{r t}{2}\right|^{\beta}}{\widetilde{\omega}(t)\left(\frac{2(m+1) \pi}{r}-t\right)^{\gamma}}\right)^{p} d t\right\}^{1 / p}=O_{x}\left((n+1)^{\gamma}\right),
\end{aligned}
$$

with $0<\gamma<\beta+\frac{1}{p}$, where $m \in\left\{0, \ldots\left[\frac{r}{2}\right]-1\right\}$. If a matrix $A$ is such that

$$
\sum_{k=0}^{\infty}(k+1)^{2} a_{n, k}=O\left((n+1)^{2}\right)
$$

and

$$
\left[\sum_{l=0}^{n} \sum_{k=l}^{r+l-1} a_{n, k}\right]^{-1}=O(1)
$$

with $r \in \mathbb{N}$ are true, then

$$
\left|\widetilde{T}_{n, A} f(x)-\widetilde{f}_{r}\left(x, \frac{\pi}{r(n+1)}\right)\right|=O_{x}\left((n+1)^{\beta+\frac{1}{p}+1} A_{n, r} \widetilde{\omega}\left(\frac{\pi}{n+1}\right)\right) .
$$

Theorem C ([3, Theorem 2]) Let $f \in L_{2 \pi}^{p}, 1<p<\infty, 0 \leq \beta<1-\frac{1}{p}$ and a function $\widetilde{\omega}$ of modulus of continuity type satisfy, for $r \in \mathbb{N}$, the conditions: (4) and (5) with $0<\gamma<\beta+\frac{1}{p}$, where $m \in\left\{0, \ldots\left[\frac{r}{2}\right]\right\}$ when $r$ is an odd or $m \in\left\{0, \ldots\left[\frac{r}{2}\right]-1\right\}$ when $r$ is an even natural number. Moreover, let $\widetilde{\omega}$ satisfy, for a natural $r \geq 2$, the conditions (6) and (7) with $0<\gamma<$ $\beta+\frac{1}{p}$, where $m \in\left\{0, \ldots\left[\frac{r}{2}\right]-1\right\}$. If a matrix $A$ is such that

$$
\sum_{k=0}^{\infty}(k+1) a_{n, k}=O(n+1)
$$

and (9) with $r \in \mathbb{N}$ are true, then

$$
\left|\widetilde{T}_{n, A} f(x)-\widetilde{f}_{r}\left(x, \frac{\pi}{r(n+1)}\right)\right|=O_{x}\left((n+1)^{\beta+\frac{1}{p}+1} A_{n, r} \widetilde{\omega}\left(\frac{\pi}{n+1}\right)\right) .
$$

In our theorems we generalize the above results considering $2 \pi / r$-periodic functions and using simpler assumptions.

In the paper $\sum_{k=a}^{b}=0$ when $a>b$. 


\section{Statement of the results}

To begin with, we will present the estimates of the quantities

$$
\left|\widetilde{T}_{n, A} f(x)-\widetilde{f}_{r}\left(x, \frac{\pi}{r(n+1)}\right)\right| \text { and }\left\|\widetilde{T}_{n, A} f(\cdot)-\widetilde{f}_{r}\left(\cdot, \frac{\pi}{r(n+1)}\right)\right\|_{L_{2 \pi / r}^{p}} .
$$

Finally, we will formulate some remarks and corollaries.

Theorem 1 Suppose that $f \in L_{2 \pi / r}^{p}, 1<p<\infty, r \in \mathbb{N}, 0 \leq \beta<1-\frac{1}{p}$ and a function $\widetilde{\omega}$ of the modulus of continuity type satisfies the conditions:

$$
\left\{\int_{0}^{\frac{\pi}{r(n+1)}}\left(\frac{t\left|\psi_{x}(t)\right|\left|\sin \frac{r t}{2}\right|^{\beta}}{\widetilde{\omega}(t)}\right)^{p} d t\right\}^{1 / p}=O_{x}\left((n+1)^{-1}\right)
$$

when $r=1$ or

$$
\left\{\int_{0}^{\frac{\pi}{r(n+1)}}\left(\frac{\left|\psi_{x}(t)\right|\left|\sin \frac{r t}{2}\right|^{\beta}}{\widetilde{\omega}(t)}\right)^{p} d t\right\}^{1 / p}=O_{x}(1)
$$

when $r \geq 2$, and

$$
\left\{\int_{\frac{\pi}{r(n+1)}}^{\frac{\pi}{r}}\left(\frac{\left|\psi_{x}(t)\right|\left|\sin \frac{r t}{2}\right|^{\beta}}{\widetilde{\omega}(t) t^{\gamma}}\right)^{p} d t\right\}^{1 / p}=O_{x}\left((n+1)^{\gamma}\right)
$$

for $r \in \mathbb{N}$ with $0<\gamma<\beta+\frac{1}{p}$. If a matrix $A$ is such that (8) and (9) are true, then

$$
\left|\widetilde{T}_{n, A} f(x)-\widetilde{f}_{r}\left(x, \frac{\pi}{r(n+1)}\right)\right|=O_{x}\left((n+1)^{\beta+\frac{1}{p}+1} A_{n, r} \widetilde{\omega}\left(\frac{\pi}{n+1}\right)\right) .
$$

Theorem 2 Suppose that $f \in L_{2 \pi / r}^{p}, 1<p<\infty, r \in \mathbb{N}, 0 \leq \beta<1-\frac{1}{p}$ and a function $\widetilde{\omega}$ of the modulus of continuity type satisfies the conditions (12) and (13) for $r \in \mathbb{N}$ with $0<\gamma<\beta+\frac{1}{p}$. If a matrix $A$ is such that (10) and (9) are true, then

$$
\left|\widetilde{T}_{n, A} f(x)-\widetilde{f}_{r}\left(x, \frac{\pi}{r(n+1)}\right)\right|=O_{x}\left((n+1)^{\beta+\frac{1}{p}+1} A_{n, r} \widetilde{\omega}\left(\frac{\pi}{n+1}\right)\right) .
$$

Remark 1 The Hölder inequality gives

$$
\begin{aligned}
\sum_{k=0}^{\infty}(k+1) a_{n, k} & =\sum_{k=0}^{\infty}(k+1) a_{n, k}^{1 / 2} a_{n, k}^{1 / 2} \leq\left[\sum_{k=0}^{\infty}(k+1)^{2} a_{n, k}\right]^{1 / 2}\left[\sum_{k=0}^{\infty} a_{n, k}\right]^{1 / 2} \\
& =\left[\sum_{k=0}^{\infty}(k+1)^{2} a_{n, k}\right]^{1 / 2}
\end{aligned}
$$

and thus the condition (8) implies (10), but the condition (12) implies (11). Therefore Theorems 1 and 2 are not comparable. 
Theorem 3 Let $f \in L_{2 \pi / r}^{p}(\widetilde{\omega})_{\beta}, 1<p<\infty, r \in \mathbb{N}$ and $0 \leq \beta<1-\frac{1}{p}$. If a matrix $A$ is such that (9) and (8) or (10) are true, then

$$
\left\|\widetilde{T}_{n, A} f(\cdot)-\widetilde{f}_{r}\left(\cdot, \frac{\pi}{r(n+1)}\right)\right\|_{L_{2 \pi / r}^{p}}=O_{x}\left((n+1)^{\beta+\frac{1}{p}+1} A_{n, r} \widetilde{\omega}\left(\frac{\pi}{n+1}\right)\right) .
$$

Corollary 1 Taking $r=1$ the conditions (11) and (13) in Theorem 1 reduce to (1) and (2). Thus we obtain the results from [2] and Theorem A [1, Theorem 8, p. 95], but in the case of [3] (Theorem B and $C$ ) we reduce the assumptions.

Next, using more natural conditions when $\beta>0$ we can formulate, without proofs, the following theorems.

Theorem 4 Suppose that $f \in L_{2 \pi / r}^{p}, 1<p<\infty, r \in \mathbb{N}, 0<\beta<1-\frac{1}{p}$. Let a function $\widetilde{\omega}$ of the modulus of continuity type satisfy the conditions:

$$
\left\{\int_{\frac{\pi}{r(n+1)}}^{\frac{\pi}{r}}\left(\frac{t^{-\gamma}\left|\psi_{x}(t)\right|\left|\sin \frac{r t}{2}\right|^{\beta}}{\widetilde{\omega}(t)}\right)^{p} d t\right\}^{1 / p}=O_{x}\left((n+1)^{\gamma-\frac{1}{p}}\right)
$$

for $\gamma \in\left(\frac{1}{p}, \frac{1}{p}+\beta\right)$ and $r \in \mathbb{N}($ instead of (13)), and

$$
\left\{\int_{0}^{\frac{\pi}{r(n+1)}}\left(\frac{t\left|\psi_{x}(t)\right|\left|\sin \frac{r t}{2}\right|^{\beta}}{\widetilde{\omega}(t)}\right)^{p} d t\right\}^{1 / p}=O_{x}\left((n+1)^{-1-\frac{1}{p}}\right)
$$

when $r=1$ or

$$
\left\{\int_{0}^{\frac{\pi}{r(n+1)}}\left(\frac{\left|\psi_{x}(t)\right|\left|\sin \frac{r t}{2}\right|^{\beta}}{\widetilde{\omega}(t)}\right)^{p} d t\right\}^{1 / p}=O_{x}\left((n+1)^{-\frac{1}{p}}\right)
$$

when $r \geq 2$ (instead of (11) and (12), respectively). If a matrix $A$ is such that (9) and (8) are true, then

$$
\left|\widetilde{T}_{n, A} f(x)-\widetilde{f}_{r}\left(x, \frac{\pi}{r(n+1)}\right)\right|=O_{x}\left((n+1)^{\beta+1} A_{n, r} \widetilde{\omega}\left(\frac{\pi}{n+1}\right)\right) .
$$

Moreover, if a function $\widetilde{\omega}$ of the modulus of continuity type and a matrix A satisfy the following conditions: (14) with $r \in \mathbb{N}$ and $\gamma \in\left(\frac{1}{p}, \frac{1}{p}+\beta\right)$, (15) with $r \in \mathbb{N}$, (9) and (10), then the estimate (16) is also true.

Theorem 5 Let $f \in L_{2 \pi / r}^{p}(\widetilde{\omega})_{\beta}$ with $1<p<\infty, r \in \mathbb{N}$ and $0<\beta<1-\frac{1}{p}$. If a matrix $A$ is such that (9) and (8) or (10) are true, then

$$
\left\|\widetilde{T}_{n, A} f(\cdot)-\widetilde{f}_{r}\left(\cdot, \frac{\pi}{r(n+1)}\right)\right\|_{L_{2 \pi / r}^{p}}=O_{x}\left((n+1)^{\beta+1} A_{n, r} \widetilde{\omega}\left(\frac{\pi}{n+1}\right)\right) .
$$

Remark 2 We note that our extra conditions (9), (8) and (10) for a lower triangular infinite matrix $A^{\circ}$ always hold. 
Corollary 2 Considering the above remarks and the obvious inequality

$$
A_{n, r} \leq r A_{n, 1} \quad \text { for } r \in \mathbb{N}
$$

our results also improve and generalize the mentioned result of Krasniqi [1].

Remark 3 We note that instead of $L_{2 \pi / r}^{p}(\widetilde{\omega})_{\beta}$ one can consider another subclass of $L_{2 \pi / r}^{p}$ generated by any function of the modulus of continuity type e.g. $\widetilde{\omega}_{x}$ such that

$$
\widetilde{\omega}_{x}(f, \delta)=\sup _{|t| \leq \delta}\left|\psi_{x}(t)\right| \leq \widetilde{\omega}_{x}(\delta)
$$

or

$$
\widetilde{\omega}_{x}(f, \delta)=\frac{1}{\delta} \int_{0}^{\delta}\left|\psi_{x}(t)\right| d t \leq \widetilde{\omega}_{x}(\delta)
$$

\section{Auxiliary results}

We begin this section by some notations from [5] and [4, Sect. 5 of Chapter II]. Let for $r=1,2, \ldots$

$$
D_{k, r}^{\circ}(t)=\frac{\sin \frac{(2 k+r) t}{2}}{2 \sin \frac{r t}{2}}, \quad \widetilde{D}_{k, r}^{\circ}(t)=\frac{\cos \frac{(2 k+r) t}{2}}{2 \sin \frac{r t}{2}}
$$

and

$$
\widetilde{D}_{k, r}(t)=\frac{\cos \frac{r t}{2}-\cos \frac{(2 k+r) t}{2}}{2 \sin \frac{r t}{2}}=\frac{\cos \frac{r t}{2}}{2 \sin \frac{r t}{2}}-\widetilde{D}_{k, r}(t) .
$$

It is clear by [4] that

$$
\widetilde{S}_{k} f(x)=-\frac{1}{\pi} \int_{-\pi}^{\pi} f(x+t) \widetilde{D}_{k, 1}(t) d t
$$

and

$$
\widetilde{T}_{n, A} f(x)=-\frac{1}{\pi} \int_{-\pi}^{\pi} f(x+t) \sum_{k=0}^{\infty} a_{n, k} \widetilde{D}_{k, 1}(t) d t .
$$

Now, we present a very useful property of the modulus of continuity.

Lemma 1 ([4]) A function $\widetilde{\omega}$ of modulus of continuity type on the interval $[0,2 \pi]$ satisfies the following condition:

$$
\delta_{2}^{-1} \widetilde{\omega}\left(\delta_{2}\right) \leq 2 \delta_{1}^{-1} \widetilde{\omega}\left(\delta_{1}\right) \quad \text { for } \delta_{2} \geq \delta_{1}>0 .
$$

Next, we present the following well-known estimates.

Lemma 2 ([4]) If $0<|t| \leq \pi$ then

$$
\left|{\widetilde{D^{\circ}}}_{k, 1}(t)\right| \leq \frac{\pi}{2|t|}, \quad\left|\widetilde{D}_{k, 1}(t)\right| \leq \frac{\pi}{|t|}
$$


and, for any real $t$, we have

$$
\left|D_{k, 1}^{\circ}(t)\right| \leq k+\frac{1}{2}, \quad\left|\widetilde{D}_{k, 1}(t)\right| \leq \frac{1}{2} k(k+1)|t|, \quad\left|\widetilde{D}_{k, 1}(t)\right| \leq k+1
$$

Lemma $3([5,6])$ Let $r \in N, l \in Z$ and $\left(a_{n}\right) \subset \mathbb{C}$. If $t \neq \frac{2 l \pi}{r}$, then for every $m \geq n$

$$
\begin{aligned}
& \sum_{k=n}^{m} a_{k} \sin k t=-\sum_{k=n}^{m}\left(a_{k}-a_{k+r}\right) \widetilde{D^{\circ}}{ }_{k, r}(t)+\sum_{k=m+1}^{m+r} a_{k}{\widetilde{D^{\circ}}}_{k,-r}(t)-\sum_{k=n}^{n+r-1} a_{k}{\widetilde{D^{\circ}}}_{k,-r}(t), \\
& \sum_{k=n}^{m} a_{k} \cos k t=\sum_{k=n}^{m}\left(a_{k}-a_{k+r}\right) D_{k, r}^{\circ}(t)-\sum_{k=m+1}^{m+r} a_{k} D_{k,-r}^{\circ}(t)+\sum_{k=n}^{n+r-1} a_{k} D_{k,-r}^{\circ}(t) .
\end{aligned}
$$

We additionally need the following estimate as a consequence of Lemma 3.

Lemma 4 Let $r \in \mathbb{N}, l \in \mathbb{Z}$ and $\left(a_{n, k}\right) \subset \mathbb{R}_{0}^{+}$for $n . k \in \mathbb{N}_{0}$. If $t \neq \frac{2 l \pi}{r}$, then

$$
\left|\frac{1}{2} \sum_{k=0}^{\infty} a_{n, k} \cos \frac{(2 k+1) t}{2}\right| \leq \frac{1}{2\left|\sin \frac{r t}{2}\right|}\left(A_{n, r}+\sum_{k=0}^{r-1} a_{n, k}\right) \leq \frac{1}{\left|\sin \frac{r t}{2}\right|} A_{n, r} .
$$

Proof By Lemma 3,

$$
\begin{aligned}
\frac{1}{2} \sum_{k=0}^{\infty} a_{n, k} \cos \frac{(2 k+1) t}{2} \\
=\frac{1}{2}\left(\sum_{k=0}^{\infty} a_{n, k} \cos k t \cos \frac{t}{2}-\sum_{k=0}^{\infty} a_{n, k} \sin k t \sin \frac{t}{2}\right) \\
=\frac{\cos \frac{t}{2}}{2}\left(\sum_{k=0}^{\infty}\left(a_{n, k}-a_{n, k+r}\right) D_{k, r}^{\circ}(t)+\sum_{k=0}^{r-1} a_{n, k} D_{k,-r}^{\circ}(t)\right) \\
\quad-\frac{\sin \frac{t}{2}}{2}\left(-\sum_{k=0}^{\infty}\left(a_{n, k}-a_{n, k+r}\right){\widetilde{D^{\circ}}}_{k, r}(t)-\sum_{k=0}^{r-1} a_{n, k}{\widetilde{D^{\circ}}}_{k,-r}(t)\right)
\end{aligned}
$$

and our inequalities follow.

We also need some special conditions which follow from the ones mentioned above.

Lemma 5 Suppose that $f \in L_{2 \pi / r}^{p}$, where $1 \leq p<\infty$ and $r \in \mathbb{N}$. If the condition (12) holds with any function $\tilde{\omega}$ of the modulus of continuity type and $\beta \geq 0$, then

$$
\left\{\int_{\frac{2(m+1) \pi}{r}-\frac{\pi}{r(n+1)}}^{\frac{2(m+1) \pi}{r}}\left(\frac{\left|\psi_{x}(t)\right|}{\widetilde{\omega}\left(\frac{2(m+1) \pi}{r}-t\right)}\right)^{p}\left|\sin \frac{r t}{2}\right|^{\beta p} d t\right\}^{\frac{1}{p}}=O_{x}(1)
$$

where $m \in\left\{0, \ldots\left[\frac{r}{2}\right]-1\right\}$.

Proof By the substitution $t=\frac{2(m+1) \pi}{r}-u$, we obtain

$$
\left\{\int_{\frac{2(m+1) \pi}{r}-\frac{\pi}{r(n+1)}}^{\frac{2(m+1) \pi}{r}}\left(\frac{\left|\psi_{x}(t)\right|}{\widetilde{\omega}\left(\frac{2(m+1) \pi}{r}-t\right)}\right)^{p}\left|\sin \frac{r t}{2}\right|^{\beta p} d t\right\}^{1 / p}
$$




$$
\begin{aligned}
& =\left\{\int_{0}^{\frac{\pi}{r(n+1)}}\left(\frac{\left|\psi_{x}\left(\frac{2(m+1) \pi}{r}-u\right)\right|}{\widetilde{\omega}(u)}\left|\sin \frac{r}{2}\left(\frac{2(m+1) \pi}{r}-u\right)\right|^{\beta}\right)^{p} d u\right\}^{1 / p} \\
& =\left\{\int_{0}^{\frac{\pi}{r(n+1)}}\left(\frac{\left|\psi_{x}(u)\right|}{\widetilde{\omega}(u)}\left|\sin \frac{r u}{2}\right|^{\beta}\right)^{p} d u\right\}^{1 / p} .
\end{aligned}
$$

Hence, by (12) our estimate follows.

Lemma 6 Suppose that $f \in L_{2 \pi / r}^{p}$, where $1 \leq p<\infty$ and $r \in \mathbb{N}$. If the condition (12) holds with any function $\widetilde{\omega}$ of the modulus of continuity type and $\beta \geq 0$, then

$$
\left\{\int_{\frac{2 m \pi}{r}}^{\frac{2 m \pi}{r}+\frac{\pi}{r(n+1)}}\left(\frac{\left|\psi_{x}(t)\right|}{\widetilde{\omega}\left(t-\frac{2 m \pi}{r}\right)}\right)^{p}\left|\sin \frac{r t}{2}\right|^{\beta p} d t\right\}^{\frac{1}{p}}=O_{x}(1),
$$

where $m \in\left\{0, \ldots\left[\frac{r}{2}\right]\right\}$.

Proof By the substitution $t=\frac{2 m \pi}{r}+u$, analogously to the above proof, we obtain

$$
\begin{aligned}
& \left\{\int_{\frac{2 m \pi}{r}}^{\frac{2 m \pi}{r}+\frac{\pi}{r(n+1)}}\left(\frac{\left|\psi_{x}(t)\right|}{\widetilde{\omega}\left(t-\frac{2 m \pi}{r}\right)}\right)^{p}\left|\sin \frac{r t}{2}\right|^{\beta p} d t\right\}^{1 / p} \\
& \quad=\left\{\int_{0}^{\frac{\pi}{r(n+1)}}\left(\frac{\left|\psi_{x}\left(\frac{2 m \pi}{r}+u\right)\right|}{\widetilde{\omega}(u)}\left|\sin \frac{r}{2}\left(\frac{2 m \pi}{r}+u\right)\right|^{\beta}\right)^{p} d u\right\}^{1 / p} \\
& \quad \leq\left\{\int_{0}^{\frac{\pi}{r(n+1)}}\left(\frac{\left|\psi_{x}(u)\right|}{\widetilde{\omega}(u)}\left|\sin \frac{r u}{2}\right|^{\beta}\right)^{p} d t\right\}^{1 / p}=O_{x}(1)
\end{aligned}
$$

and we have the desired estimate.

Now, we formulate another two lemmas without proofs. We can prove them in the same way as Lemmas 5 and 6, respectively.

Lemma 7 Suppose that $f \in L_{2 \pi / r}^{p}$, where $1 \leq p<\infty$ and $r \in \mathbb{N}$. If the condition (13) holds with any function $\widetilde{\omega}$ of the modulus of continuity type and $\gamma, \beta \geq 0$, then

$$
\left\{\int_{\frac{2(m+1) \pi}{r}-\frac{\pi}{r}}^{\frac{2(m+1) \pi}{r(n+1)}}\left(\frac{\left|\psi_{x}(t)\right|\left|\sin \frac{r t}{2}\right|^{\beta}}{\widetilde{\omega}(t)\left(\frac{2(m+1) \pi}{r}-t\right)^{\gamma}}\right)^{p} d t\right\}^{1 / p}=O_{x}\left((n+1)^{\gamma}\right),
$$

where $m \in\left\{0, \ldots\left[\frac{r}{2}\right]-1\right\}$.

Lemma 8 Suppose that $f \in L_{2 \pi / r}^{p}$, where $1 \leq p<\infty$ and $r \in \mathbb{N}$. If the condition (13) holds with any function $\tilde{\omega}$ of the modulus of continuity type and $\gamma, \beta \geq 0$, then

$$
\left\{\int_{\frac{2 m \pi}{r}+\frac{\pi}{r(n+1)}}^{\frac{2 m \pi}{r}+\frac{\pi}{r}}\left(\frac{\left|\psi_{x}(t)\right|\left|\sin \frac{r t}{2}\right|^{\beta}}{\widetilde{\omega}(t)\left(t-\frac{2 m \pi}{r}\right)^{\gamma}}\right)^{p} d t\right\}^{1 / p}=O_{x}\left((n+1)^{\gamma}\right),
$$

where $m \in\left\{0, \ldots\left[\frac{r}{2}\right]\right\}$. 


\section{Proofs of theorems}

\subsection{Proof of Theorem 1}

It is clear that for odd $r$

$$
\begin{aligned}
& \widetilde{T}_{n, A} f(x)-\widetilde{f}_{r}\left(x, \frac{\pi}{r(n+1)}\right) \\
&=-\frac{1}{\pi} \int_{0}^{\pi} \psi_{x}(t) \sum_{k=0}^{\infty} a_{n, k} \widetilde{D}_{k, 1}(t) d t \\
&+\frac{1}{\pi}\left(\sum_{m=0}^{[r / 2]-1} \int_{\frac{2 m \pi}{r}+\frac{\pi}{r(n+1)}}^{\frac{2(m+1) \pi}{r}-\frac{\pi}{r(n+1)}}+\int_{\frac{2[r / 2] \pi}{r}+\frac{\pi}{r(n+1)}}^{\frac{(2[r / 2]+1) \pi}{r}}\right) \psi_{x}(t) \frac{1}{2} \cot \frac{t}{2} d t \\
&=-\frac{1}{\pi}\left(\int_{0}^{\frac{\pi}{r(n+1)}}+\sum_{m=1}^{[r / 2]} \int_{\frac{2 m \pi}{r}}^{\frac{2 m \pi}{r}+\frac{\pi}{r(n+1)}}+\sum_{\frac{[r / 2]-1}{m=0}}^{\frac{2(m+1) \pi}{r}} \int_{\frac{2(m+1) \pi}{r}-\frac{\pi}{r(n+1)}}\right) \\
& \times \psi_{x}(t) \sum_{k=0}^{\infty} a_{n, k} \widetilde{D}_{k, 1}(t) d t \\
&+\frac{1}{\pi}\left(\sum_{m=0}^{[r / 2]} \int_{\frac{2 m \pi}{r}+\frac{\pi}{r(n+1)}}^{\frac{2(m+1) \pi}{r}}+\sum_{m=0}^{[r / 2]-1} \int_{\frac{(2 m+1) \pi}{r}}^{\frac{2(m+1) \pi}{r}-\frac{\pi}{r(n+1)}} \psi_{x}(t) \sum_{k=0}^{\infty} a_{n, k} \widetilde{D}_{k, 1}^{\circ}(t) d t\right. \\
&= I_{0}(x)+I_{1}(x)+I_{2}(x)+I_{3}(x)+I_{4}(x)
\end{aligned}
$$

and for even $r$

$$
\begin{aligned}
& \widetilde{T}_{n, A} f(x)-\widetilde{f}_{r}\left(x, \frac{\pi}{r(n+1)}\right) \\
&=-\frac{1}{\pi} \int_{0}^{\pi} \psi_{x}(t) \sum_{k=0}^{\infty} a_{n, k} \widetilde{D}_{k, 1}(t) d t+\frac{1}{\pi} \sum_{m=0}^{[r / 2]-1} \int_{\frac{2 m \pi}{r}+\frac{\pi}{r(n+1)}}^{\frac{2(m+1) \pi}{r}-\frac{\pi}{r(n+1)}} \psi_{x}(t) \frac{1}{2} \cot \frac{t}{2} d t \\
&=-\frac{1}{\pi}\left(\int_{0}^{\frac{\pi}{r(n+1)}}+\sum_{m=1}^{[r / 2]-1} \int_{\frac{2 m \pi}{r}}^{\frac{2 m \pi}{r}+\frac{\pi}{r(n+1)}}+\sum_{m=0}^{[r / 2]-1} \int_{\frac{2(m+1) \pi}{r}-\frac{\pi}{r(n+1)}}^{\frac{2(m+1) \pi}{r}}\right) \\
& \times \psi_{x}(t) \sum_{k=0}^{\infty} a_{n, k} \widetilde{D}_{k, 1}(t) d t \\
&+\frac{1}{\pi}\left(\sum_{m=0}^{[r / 2]-1} \int_{\frac{2 m \pi}{r}+\frac{\pi}{r(n+1)}}^{\frac{(2 m+1) \pi}{r}}+\sum_{m=0}^{[r / 2]-1} \int_{\frac{(2 m+1) \pi}{r}}^{\left.\frac{2(m+1) \pi}{r}-\frac{\pi}{r(n+1)}\right)}\right. \\
& \times \psi_{x}(t) \sum_{k=0}^{\infty} a_{n, k} \widetilde{D}_{k, 1}^{\circ}(t) d t \\
&= I_{0}(x)+I_{1}^{\prime}(x)+I_{2}(x)+I_{3}^{\prime}(x)+I_{4}(x),
\end{aligned}
$$

whence

$$
\begin{aligned}
& \left|\widetilde{T}_{n, A} f(x)-\widetilde{f}_{r}\left(x, \frac{\pi}{r(n+1)}\right)\right| \\
& \quad \leq\left|I_{0}(x)\right|+\left|I_{1}(x)\right|+\left|I_{1}^{\prime}(x)\right|+\left|I_{2}(x)\right|+\left|I_{3}(x)\right|+\left|I_{3}^{\prime}(x)\right|+\left|I_{4}(x)\right| .
\end{aligned}
$$


Next, using Lemma 2, (8), the Hölder inequality with $p>1$ and $q=\frac{p}{p-1}$ and (11) when $r=1$ or (12) when $r \geq 2$ we get

$$
\begin{aligned}
& \left|I_{0}(x)\right| \\
& \quad=O\left((n+1)^{2}\right) \int_{0}^{\frac{\pi}{r(n+1)}} t\left|\psi_{x}(t)\right| d t \\
& \quad \leq O\left((n+1)^{2}\right)\left\{\int_{0}^{\frac{\pi}{r(n+1)}}\left(\frac{t\left|\psi_{x}(t)\right|}{\widetilde{\omega}(t)}\right)^{p} \sin ^{\beta p} \frac{r t}{2} d t\right\}^{1 / p}\left\{\int_{0}^{\frac{\pi}{r(n+1)}}\left(\frac{\widetilde{\omega}(t)}{\sin ^{\beta} \frac{r t}{2}}\right)^{q} d t\right\}^{\frac{1}{q}} \\
& \quad \leq O\left((n+1)^{2}\right) O_{x}\left((n+1)^{-1}\right) \widetilde{\omega}\left(\frac{\pi}{r(n+1)}\right)\left\{\int_{0}^{\frac{\pi}{r(n+1)}}\left(\frac{\pi}{r t}\right)^{\beta q} d t\right\}^{\frac{1}{q}} \\
& \quad=O_{x}((n+1)) \widetilde{\omega}\left(\frac{\pi}{r(n+1)}\right)\left(\frac{\pi}{r(n+1)}\right)^{\frac{1}{q}-\beta}=O_{x}\left((n+1)^{\beta+\frac{1}{p}}\right) \widetilde{\omega}\left(\frac{\pi}{n+1}\right),
\end{aligned}
$$

for $0 \leq \beta<1-\frac{1}{p}$. We note that applying the condition (9) we have

$$
\begin{aligned}
{\left[(n+1) A_{n, r}\right]^{-1} } & =\left[\sum_{l=0}^{n} A_{n, r}\right]^{-1} \leq\left[\sum_{l=0}^{n} \sum_{k=l}^{\infty}\left|a_{n, k}-a_{n, k+r}\right|\right]^{-1} \\
& \leq\left[\sum_{l=0}^{n}\left|\sum_{k=l}^{\infty}\left(a_{n, k}-a_{n, k+r}\right)\right|\right]^{-1}=\left[\sum_{l=0}^{n} \sum_{k=l}^{r+l-1} a_{n, k}\right]^{-1}=O(1),
\end{aligned}
$$

whence

$$
\left|I_{0}(x)\right|=O_{x}\left((n+1)^{1+\beta+\frac{1}{p}} A_{n, r} \widetilde{\omega}\left(\frac{\pi}{n+1}\right)\right) .
$$

By Lemma 2

$$
\begin{aligned}
& \left|I_{1}(x)\right|+\left|I_{1}^{\prime}(x)\right|+\left|I_{2}(x)\right| \\
& \leq \frac{1}{\pi}\left(\sum_{m=1}^{[r / 2]} \int_{\frac{2 m \pi}{r}}^{\frac{2 m \pi}{r}+\frac{\pi}{r(n+1)}}+\sum_{m=0}^{[r / 2]-1} \int_{\frac{2(m+1) \pi}{r}-\frac{\pi}{r(n+1)}}^{\frac{2(m+1) \pi}{r}}\right) \frac{\left|\psi_{x}(t)\right|}{t} d t \\
& \quad \leq \frac{1}{\pi}\left(\sum_{m=1}^{[r / 2]} \int_{\frac{2 m \pi}{r}}^{\frac{2 m \pi}{r}+\frac{\pi}{r(n+1)}}+\sum_{m=0}^{[r / 2]-1} \int_{\frac{2(m+1) \pi}{r}-\frac{\pi}{r(n+1)}}^{\frac{2(m+1) \pi}{r}}\right) \frac{\left|\psi_{x}(t)\right|}{\pi / r} d t
\end{aligned}
$$

and using the Hölder inequality with $p>1$ and $q=\frac{p}{p-1}$

$$
\begin{aligned}
& \left|I_{1}(x)\right|+\left|I_{1}^{\prime}(x)\right|+\left|I_{2}(x)\right| \\
& \leq O_{x}(1) \sum_{m=1}^{[r / 2]}\left[\int_{\frac{2 m \pi}{r}}^{\frac{2 m \pi}{r}+\frac{\pi}{r(n+1)}}\left(\frac{\left|\psi_{x}(t)\right| \sin ^{\beta} \frac{r t}{2}}{\widetilde{\omega}\left(t-\frac{2 m \pi}{r}\right)}\right)^{p} d t\right]^{\frac{1}{p}} \\
& \quad \times\left[\int_{\frac{2 m \pi}{r}}^{\frac{2 m \pi}{r}+\frac{\pi}{r(n+1)}}\left(\frac{\widetilde{\omega}\left(t-\frac{2 m \pi}{r}\right)}{\sin ^{\beta} \frac{r t}{2}}\right)^{q} d t\right]^{\frac{1}{q}} \\
& \quad+O_{x}(1) \sum_{m=1}^{[r / 2]-1}\left[\int_{\frac{2(m+1) \pi}{r}-\frac{\pi}{r(n+1)}}^{\frac{2(m+1) \pi}{\sigma}}\left(\frac{\left|\psi_{x}(t)\right| \sin ^{\beta} \frac{r t}{2}}{\widetilde{\omega}\left(\frac{2(m+1) \pi}{r}-t\right)}\right)^{p} d t\right]^{\frac{1}{p}}
\end{aligned}
$$




$$
\times\left[\int_{\frac{2(m+1) \pi}{r}-\frac{\pi}{r(n+1)}}^{\frac{2(m+1) \pi}{r}}\left(\frac{\widetilde{\omega}\left(\frac{2(m+1) \pi}{r}-t\right)}{\sin ^{\beta} \frac{r t}{2}}\right)^{q} d t\right]^{\frac{1}{q}}
$$

Hence, by Lemmas 5 and 6 with (12) and (9),

$$
\begin{aligned}
& \left|I_{1}(x)\right|+\left|I_{1}^{\prime}(x)\right|+\left|I_{2}(x)\right| \\
& \quad=O_{x}(1) \widetilde{\omega}\left(\frac{\pi}{r(n+1)}\right)\left[\int_{0}^{\frac{\pi}{r(n+1)}}\left(\frac{1}{\sin ^{\beta} \frac{r t}{2}}\right)^{q} d t\right]^{\frac{1}{q}} \\
& \quad=O_{x}\left((n+1)^{\beta-\frac{1}{q}}\right) \widetilde{\omega}\left(\frac{\pi}{n+1}\right)=O_{x}\left((n+1)^{\beta+\frac{1}{p}} A_{n, r} \widetilde{\omega}\left(\frac{\pi}{n+1}\right)\right),
\end{aligned}
$$

for $0 \leq \beta<1-\frac{1}{p}$.

In the case of the last integrals, applying Lemma 4 we obtain

$$
\begin{aligned}
& \left|I_{3}(x)\right|+\left|I_{3}^{\prime}(x)\right|+\left|I_{4}(x)\right| \\
& \quad \leq \frac{1}{\pi}\left(\sum_{m=0}^{[r / 2]} \int_{\frac{2 m \pi}{r}+\frac{\pi}{r(n+1)}}^{\frac{(2 m+1) \pi}{r}}+\sum_{m=0}^{[r / 2]-1} \int_{\frac{(2 m+1) \pi}{r}}^{\frac{2(m+1) \pi}{r}-\frac{\pi}{r(n+1)}}\right) \frac{\left|\psi_{x}(t)\right|}{\left|\sin \frac{t}{2} \sin \frac{r t}{2}\right|} A_{n, r} d t .
\end{aligned}
$$

Using the estimates $\left|\sin \frac{t}{2}\right| \geq \frac{|t|}{\pi}$ for $t \in[0, \pi],\left|\sin \frac{r t}{2}\right| \geq \frac{r t}{\pi}-2 m$ for $t \in\left[\frac{2 m \pi}{r}+\frac{\pi}{r(n+1)}, \frac{(2 m+1) \pi}{r}\right]$, where $m \in\{0, \ldots,[r / 2]\}$ and $\left|\sin \frac{r t}{2}\right| \geq 2(m+1)-\frac{r t}{\pi}$ for $t \in\left[\frac{(2 m+1) \pi}{r}, \frac{2(m+1) \pi}{r}-\frac{\pi}{r(n+1)}\right]$, where $m \in\{0, \ldots,[r / 2]-1\}$, we obtain

$$
\begin{aligned}
& \left|I_{3}(x)\right|+\left|I_{3}^{\prime}(x)\right|+\left|I_{4}(x)\right| \\
& \leq A_{n, r} \sum_{m=0}^{[r / 2]} \int_{\frac{2 m \pi}{r}+\frac{\pi}{r(n+1)}}^{\frac{(2 m+1) \pi}{r}} \frac{\left|\psi_{x}(t)\right|}{\frac{r t}{\pi}\left(t-\frac{2 m \pi}{r}\right)} d t \\
& \quad+A_{n, r} \sum_{m=0}^{[r / 2]-1} \int_{\frac{(2 m+1) \pi}{r}}^{\frac{2(m+1) \pi}{r}-\frac{\pi}{r(n+1)}} \frac{\left|\psi_{x}(t)\right|}{\frac{r t}{\pi}\left[\frac{2(m+1) \pi}{r}-t\right]} d t .
\end{aligned}
$$

By the Hölder inequality with $p>1$ and $q=\frac{p}{p-1}$ we have

$$
\begin{aligned}
\left|I_{3}(x)\right| & +\left|I_{3}^{\prime}(x)\right|+\left|I_{4}(x)\right| \\
\leq & \frac{\pi}{r} A_{n, r} \sum_{m=0}^{[r / 2]}\left[\int_{\frac{2 m \pi}{r}+\frac{\pi}{r(n+1)}}^{\frac{2 m \pi}{r}+\frac{\pi}{r}}\left(\frac{\left|\psi_{x}(t)\right|}{\widetilde{\omega}(t)\left(t-\frac{2 m \pi}{r}\right)^{\gamma}}\left|\sin \frac{r t}{2}\right|^{\beta}\right)^{p} d t\right]^{\frac{1}{p}} \\
& \times\left[\int_{\frac{2 m \pi}{r}+\frac{\pi}{r(n+1)}}^{\frac{2 m \pi}{r}+\frac{\pi}{r}}\left(\frac{\widetilde{\omega}(t)\left(t-\frac{2 m \pi}{r}\right)^{\gamma}}{t\left(t-\frac{2 m \pi}{r}\right)\left|\sin \frac{r t}{2}\right|^{\beta}}\right)^{q} d t\right]^{\frac{1}{q}} \\
+ & \frac{\pi}{r} A_{n, r} \sum_{m=0}^{[r / 2]-1}\left[\int_{\frac{2(m+1) \pi}{r}-\frac{\pi}{r}}^{\frac{2(m+1) \pi}{r}-\frac{\pi}{r(n+1)}}\left(\frac{\left|\psi_{x}(t)\right|}{\widetilde{\omega}(t)\left(\frac{2(m+1) \pi}{r}-t\right)^{\gamma}}\left|\sin \frac{r t}{2}\right|^{\beta}\right)^{p} d t\right]^{\frac{1}{p}} \\
& \times\left[\int_{\frac{2(m+1) \pi}{r}-\frac{\pi}{r}}^{\frac{2(m+1) \pi}{r(n+1)}}\left(\frac{\widetilde{\omega}(t)\left(\frac{2(m+1) \pi}{r}-t\right)^{\gamma}}{t\left(\frac{2(m+1) \pi}{r}-t\right)\left|\sin \frac{r t}{2}\right|^{\beta}}\right)^{q} d t\right]^{\frac{1}{q}} \cdot
\end{aligned}
$$


Further, using Lemmas 7 and 8 with (13) and Lemma 1 we get

$$
\begin{aligned}
& \left|I_{3}(x)\right|+\left|I_{3}^{\prime}(x)\right|+\left|I_{4}(x)\right| \\
& \leq O_{x}(1) A_{n, r} \sum_{m=0}^{[r / 2]}(n+1)^{\gamma}\left[\int_{\frac{2 m \pi}{r}+\frac{\pi}{r(n+1)}}^{\frac{2 m \pi}{r}+\frac{\pi}{r}}\left(\frac{\widetilde{\omega}(t)\left(t-\frac{2 m \pi}{r}\right)^{\gamma}}{t\left(t-\frac{2 m \pi}{r}\right)\left|\sin \frac{r t}{2}\right|^{\beta}}\right)^{q} d t\right]^{\frac{1}{q}} \\
& +O_{x}(1) A_{n, r} \sum_{m=0}^{[r / 2]-1}(n+1)^{\gamma}\left[\int_{\frac{2(m+1) \pi}{r}-\frac{\pi}{r}}^{\frac{2(m+1) \pi}{r(n+1)}}\left(\frac{\widetilde{\omega}(t)\left(\frac{2(m+1) \pi}{r}-t\right)^{\gamma}}{t\left(\frac{2(m+1) \pi}{r}-t\right)\left|\sin \frac{r t}{2}\right|^{\beta}}\right)^{q} d t\right]^{\frac{1}{q}} \\
& =O_{x}(1) A_{n, r}\left[\sum_{m=0}^{[r / 2]}(n+1)^{\gamma}\left\{\int_{\frac{\pi}{r(n+1)}}^{\frac{\pi}{r}}\left(\frac{\widetilde{\omega}\left(t+\frac{2 m \pi}{r}\right) t^{\gamma-1}}{\left(t+\frac{2 m \pi}{r}\right)\left|\sin \frac{r t}{2}\right|^{\beta}}\right)^{q} d t\right\}^{\frac{1}{q}}\right. \\
& \left.+\sum_{m=0}^{[r / 2]-1}(n+1)^{\gamma}\left\{\int_{\frac{\pi}{r(n+1)}}^{\frac{\pi}{r}}\left(\frac{\widetilde{\omega}\left(\frac{2(m+1) \pi}{r}-t\right) t^{\gamma-1}}{\left(\frac{2(m+1) \pi}{r}-t\right)\left|\sin \frac{r t}{2}\right|^{\beta}}\right)^{q} d t\right\}^{\frac{1}{q}}\right] \\
& =O_{x}(1) A_{n, r}(n+1)^{\gamma}\left\{\int_{\frac{\pi}{r(n+1)}}^{\frac{\pi}{r}}\left(\frac{\widetilde{\omega}(t) t^{\gamma-1}}{t\left|\sin \frac{r t}{2}\right|^{\beta}}\right)^{q} d t\right\}^{\frac{1}{q}} \\
& =O_{x}(1) A_{n, r}(n+1)^{1+\gamma} \widetilde{\omega}\left(\frac{\pi}{r(n+1)}\right)\left(\int_{\frac{\pi}{r(n+1)}}^{\frac{\pi}{r}} t^{(\gamma-1-\beta) q} d t\right)^{\frac{1}{q}} \\
& =O_{x}(1) A_{n, r}(n+1)^{1+\gamma} \widetilde{\omega}\left(\frac{\pi}{r(n+1)}\right)(n+1)^{1+\beta-\gamma-\frac{1}{q}} \\
& =O_{x}\left((n+1)^{1+\beta+\frac{1}{p}} A_{n, r} \widetilde{\omega}\left(\frac{\pi}{(n+1)}\right)\right)
\end{aligned}
$$

for $0<\gamma<\beta+\frac{1}{p}$.

Collecting the partial estimates our statement follows.

\subsection{Proof of Theorem 2}

The proof is the same as above, but for estimate of $\left|I_{0}(x)\right|$ we only used the inequality $\left|\widetilde{D}_{k, 1}(t)\right| \leq k+1$ from Lemma 2 , and the condition (10) instead of (8).

\subsection{Proof of Theorem 3}

We note that for the estimate of $\left\|\widetilde{T}_{n, A} f(\cdot)-\widetilde{f}_{r}\left(\cdot, \frac{\pi}{(n+1)}\right)\right\|_{L_{2 \pi}^{p}}$ we need the conditions on $\widetilde{\omega}$ from the assumptions of Theorems 1 or 2 . These conditions always hold with $\|\psi(t)\|_{L_{2 \pi / r}^{p}}$ instead of $\left|\psi_{x}(t)\right|$ and thus the desired result follows.

\section{Competing interests}

The authors declare that they have no competing interests.

\section{Authors' contributions}

MK, W $Ł$ and BS contributed equally in all stages to the writing of the paper. All authors read and approved the final manuscript.

\section{Publisher's Note}

Springer Nature remains neutral with regard to jurisdictional claims in published maps and institutional affiliations. 
References

1. Krasniqi, X.Z.: Slight extensions of some theorems on the rate of pointwise approximation of functions from some subclasses of $L^{P}$. Acta Comment. Univ. Tartu Math. 17, 89-101 (2013)

2. Łenski, W., Szal, B.: Approximation of functions belonging to the class $L^{P}(\omega)$ by linear operators. Acta Comment. Univ. Tartu Math. 13, 11-24 (2009)

3. Łenski, W., Szal, B.: Pointwise approximation of modified conjugate functions by matrix operators of their Fourier series Proc. Est. Acad. Sci. 67(1), 50-60 (2018)

4. Zygmund, A.: Trigonometric Series. Cambridge University Press, Cambridge (2002)

5. Szal, B.: On L-convergence of trigonometric series. J. Math. Anal. Appl. 373, 449-463 (2011)

6. Szal, B.: A new class of numerical sequences and its applications to uniform convergence of sine series. Math. Nachr. 284(14-15), 1985-2002 (2011)

Submit your manuscript to a SpringerOpen ${ }^{\circ}$ journal and benefit from:

- Convenient online submission

- Rigorous peer review

- Open access: articles freely available online

- High visibility within the field

- Retaining the copyright to your article

Submit your next manuscript at $\gg$ springeropen.com 